\title{
AVALIAÇÃO DO GANHO DE PESO DE NOVILHAS OVARIECTOMIZADAS POR TÉCNICA TRANSVAGINAL
}

\author{
Analysis of the Weight Profit of Heifers Castrated by Transvaginal Technique
}

\author{
Ciro Meirelles \\ Médico Veterinário, Curitiba - PR. e-mail: ciromeirelles@bol.com.br \\ Carlos Francisco Bueno Junior \\ Médico Veterinário, Curitiba - PR. e-mail: cfbuenojunior@uol.com.br \\ Luiz Ernandes Kozicki \\ Médico Veterinário, Ph.D., Prof. da PUCPR, Curitiba - PR. e-mail: kozicki@ufpr.br \\ Romildo Romualdo Weiss \\ Médico Veterinário, Dr., Prof. da UFPR, Curitiba - PR. e-mail: rrweiss@bol.com.br \\ Márcio Saporski Segui \\ Médico Veterinário, M. Sc., Prof. da PUCPR, Curitiba - PR. e-mail: msegui@uol.com.br
}

\section{Resumo}

Foram selecionadas 62 novilhas, 3/4 sangue britânico e 1/4 sangue zebuíno, entre 14 e 16 meses de idade; 23 foram ovariectomizadas bilateralmente (Grupo Tratado) e os 39 animais restantes formaram o grupo controle. Todos os animais foram pesados nos dias 0, 4, 24, 47 e 56 após o procedimento da ovariectomia. Para o procedimento, foi utilizada a via transvaginal com aplicação de um anel de látex no pedículo ovariano das novilhas. Foi analisado o ganho de peso médio diário (GPMD) e o peso médio (PM) das novihas após o confinamento de 56 dias, evidenciando um ganho superior no grupo de novilhas controle em relação ao grupo ovariectomizado.

Palavras-chave: Castração; Novilha; Peso.

\section{Abstract}

Sixty-two crossbred heifers (14-16 months of age) with 3/4 british blood and $1 / 4$ zebu blood were used in this experiment. Twenty-three heifers were bilaterally spayed (treated group) and thirty-nine remaining animals formed the control group. All the animals were weighed on days $0,4,24,47$ and 56 after the procedure of ovariectomy. For the procedure it was used the transvaginal way using a latex ring in the ovarian pedicle of the heifers. It was analyzed the average daily gain (GPMD) and the average weight (PM) of feedlot heifers during 56 days, showing an increase on weight gain of the control group higher than in the treated group.

Keywords: Castration; Heifer; Weight. 


\section{INTRODUÇÃO}

O lucro obtido na bovinocultura de corte certamente é dependente do rendimento da carcaça dos animais destinados ao abate. Um aspecto discutido na produção de bovinos é o menor rendimento da carcaça das fêmeas em relação aos machos. Por esse motivo, essa categoria animal é discriminada pelas indústrias frigoríficas em virtude do menor retorno financeiro e conseqüente menor preço pago ao produtor (CROUSE, 1989; ZOBELL, 1993). Quando se trata da utilização de fêmeas para o abate, os sistemas de produção de bovinos de corte devem prever técnicas de manejo diferenciadas para estas fêmeas, já que a prenhez, nesse caso, é indesejada, assim como o estresse causado pela presença de fêmeas em estro junto ao grupo de animais.

$\mathrm{Na}$ tentativa de contornar essa situação, Brown (1984) e Drost (1992) sugeriram a eliminação do estro naturalmente apresentado pelas fêmeas por meio de técnicas como a ovariectomia. Silva et al. (2004) propuseram a aplicação do anel de látex no pedículo ovariano. Horton (1979), Turin et al. (1997) e Silva (2001) sugeriram a utilização do dispositivo Intra-Uterino (DIU) e Reiling (1996) alvitrou o tratamento com acetato de melengestrol.

Segundo Habermehl (1993), fêmeas castradas são mais valorizadas, pois desenvolvem consideravelmente a porção anterior do corpo, com bom aspecto fenotípico. De acordo com O'Brien et al. (1968) e Young et al. (1969), o estro diminui a conversão alimentar e o ganho de peso, contribuindo para a baixa produtividade das fêmeas bovinas não castradas. Para Fleck et al. (1980), Bailey et al. (1991) e Lynch et al. (1997), os ciclos reprodutivos interferem no ganho de peso de novilhas destinadas ao abate, justificando a supressão da atividade ovariana.

A ovariectomia também pode ser usada em fêmeas de descarte, ou seja, animais que não se enquadram no padrão racial desejado, como vacas velhas ou com problemas reprodutivos, além de novilhas com carcterísticas reprodutivas não desejadas. Essa prática é usada para valorizar os animais que seriam vendidos por um valor abaixo do mercado. Segundo Berge e Westhues (1975), a castração de fêmeas bovinas também é indicada para facilitar o manejo do rebanho, incrementar o rendimento lácteo e tratar casos de ninfomania. Para Brown (1984), as vantagens da ovariectomia em vacas residem no fato de se evitar surpresas desagradáveis no lote de invernada, decorrentes de coberturas não desejadas, o que facilita a eliminação de animais indesejáveis do rebanho e possibilita imprimir um desenvolvimento mais rápido aos bezerros da última parição, pelo prolongamento da lactação. Hill (1983) observou, ainda, uma considerável praticabilidade de manejo, pois fêmeas e machos podem permanecer na mesma pastagem ou confinamento.

Apesar das vantagens da esterilização cirúrgica de novilhas e vacas, trata-se de um procedimento ainda pouco difundido, pois requer técnicas e equipamentos especializados. O objetivo do presente trabalho foi avaliar o ganho de peso de novilhas ovariectomizadas, bem como avaliar a técnica transvaginal mediante utilização de anel de látex no pedículo ovariano para supressão do estro.

\section{MATERIAL E MÉTODOS}

Para o experimento, foram selecionadas 62 novilhas, 3/4 sangue britânico e $1 / 4$ sangue zebuíno, entre 14 e 16 meses de idade, de uma fazenda localizada em Palmeira (PR). O experimento foi realizado de julho a setembro de 2006. Todas as fêmeas foram submetidas a um regime alimentar à base de silagem de milho (ad libitum), $1300 \mathrm{~g}$ de ração peletizada de engorda, $500 \mathrm{~g}$ de caroço de algodão e sal mineral (ad libitum), em confinamento por 56 dias após o experimento até serem abatidas.

Dos 62 animais pesquisados, 23 foram ovariectomizados bilateralmente (Grupo Tratado) e os 39 animais restantes formaram o grupo controle (animais não ovariectomizados). Para o procedimento, foi utilizada a técnica de castração transvaginal com aplicação de um anel de látex, comumente utilizado para castração e descola de cordeiros, aplicado no pedículo ovariano das novilhas. A técnica consiste no acesso à cavidade abdominal por uma incisão na parede do vestíbulo vaginal, dorsalmente à cérvixe. Posteriormente, um equipamento cilíndrico de aço inox é introduzido na incisão e o ovário é introduzido no orifício do cilindro, permitindo a colocação do anel de látex no pedículo ovariano. 
Todos os animais foram pesados no dia do experimento (dia 0) e nos dias 4, 24, 47 e 56 após a ovariectomia. O ganho de peso médio diário e a média de peso dos dois grupos foram avaliados. O tratamento estatístico utilizado para avaliar as médias foi o teste $t$ de Student.

\section{RESULTADOS E DISCUSSÃO}

Os resultados do ganho de peso médio e ganho de peso médio diário estão apresentados na Tabela 1. O ganho de peso médio diário (GPMD), no final do experimento, do grupo controle, foi de 1,332 $\mathrm{kg}$ contra 1,118 kg do grupo ovariectomizado. A maior variação entre as médias ocorreu na pesagem do dia 4, na qual o grupo de animais ovariectomizados teve um GPMD negativo de $-0,141 \mathrm{~kg}$, ao passo que o grupo controle apresentou crescimento médio de 3,388 kg. Nas pesagens dos dias 24 e 56, o grupo tratado acusou média superior, porém a média final do período em confinamento (56 dias) foi estatisticamente favorável ao grupo controle $(\mathrm{P}<0,05)$. O ganho de peso médio do grupo controle foi superior ao do grupo tratado, 74,62 kg e $66,17 \mathrm{~kg}$, respectivamente.

TABELA 1 - Peso médio e ganho de peso médio diário nos diferentes dias após a ovariectomia Table 1 - Average weight and profit of daily average weight in the different days after ovariectomy

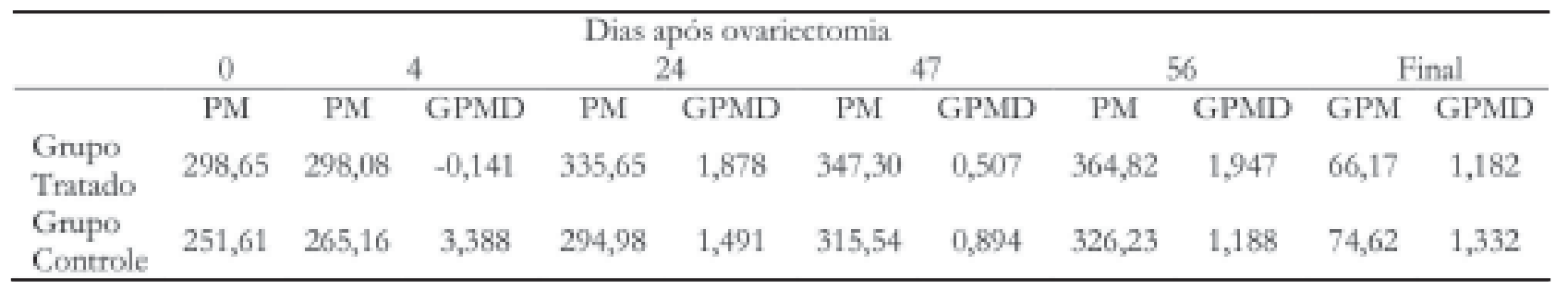

$\mathrm{PM}=$ média de peso dos animias

GPMD = ganho de peso médio diário

$\mathrm{GPM}=$ ganho de peso médio

O baixo ganho de peso apresentado pelos animais logo após a ovariectomia provavelmente está relacionado com manifestações de dor que as novilhas tratadas apresentaram em resposta à técnica e conseqüente diminuição da ingestão de alimentos, com posterior recuperação do peso, porém não suficiente para igualar-se ao grupo controle. Outro fato a ser realçado é o pouco tempo ocorrido entre o tratamento e o abate dos animais. É possível que um período maior que 56 dias de confinamento possa ter um efeito positivo no ganho de peso e rendimento de carcaça dos animais tratados.

Experimentos semelhantes, conduzidos por Silva et al. (2006), não demonstraram diferenças significativas no GPMD dos animais. As bezerras castradas por meio cirúrgico via abdominal obtiveram ganho de peso aos 30 dias após o procedimento, sem, no entanto, apresentarem diferença significativa em comparação ao grupo controle. Apesar de não significativo, o rendimento de carcaça das fêmeas castradas foi superior ao grupo controle. Trabalhos realizados por Farias Júnior (2000) demonstraram resultados semelhantes ao encontrado no experimento em questão. Novilhas com estro interrompido após aplicação do anel de látex tiveram perda de peso após o procedimento. Esse fato foi atribuído ao estresse causado pela compressão das terminações nervosas do pedículo ovariano. Para ZoBell et al. (1993), um incremento no ganho de peso diário é possível somente se as novilhas ovariectomizadas forem submetidas a tratamentos hormonais. Entretanto, Turin et al. (1997) demonstraram resultados 25,5\% superiores em fêmeas Bos taurus taurus com interrupção de estro com dispositivos intra-uterinos (DIU). 
A discrepância entre os resultados pode estar relacionada com diferenças entre o manejo adotado, raça dos animais e método de interrupção do estro, entre outros. Turin et al. (1997) citam o ganho de peso significativo como resultado da degeneração ovariana provocada pela implantação do DIU e, conseqüente, aumento na produção de testosterona. No entanto, Silva et al. (2005) questionam esses resultados com base nos dados de Saba, Cunningham e Millar (1975), que obtiveram valores inferiores desse hormônio, quando coletado na veia ovariana após a aplicação do DIU. Segundo Pineda (1989) e Allrich (1994), a degeneração ovariana não permitiria a aromatização da testosterona pelas células foliculares, justificando a não alteração no rendimento de carcaça e a incorporação de massa muscular.

Nesse experimento é esperada a degeneração ovariana após a aplicação do anel de látex, confirmado em experimentos anteriores não publicados, já que não houve manifestação de estro durante o confinamento, portanto, corrobora com o que foi postulado por Pineda (1989) e Allrich (1994) uma vez que os resultados de ganho de peso foram inferiores.

\section{CONCLUSÕES}

Apesar de os resultados não satisfatórios para ganho de peso nas novilhas, a técnica de castração transvaginal com aplicação de anel de látex no pedículo ovariano demonstrou ser eficaz para a interrupção do estro, além de ser uma técnica de fácil aplicação, baixo custo e rápida execução, podendo ser aplicada para outras finalidades. Entretanto, não fica aqui recomendado o uso da técnica em novilhas pelo sofrimento que lhes é causado no procedimento de passagem da mão via transvaginal devido ao tamanho reduzido do seu trato reprodutivo. Mais estudos seriam interessantes para verificar o efeito da ovariectomia pela técnica transvaginal no ganho de peso, rendimento e conformação de carcaça de fêmeas com pelo menos uma parição.

\section{REFERÊNCIAS}

ALLRICH, R. D. Endocrine and neural control of oestrus in dairy cows. Journal of Dairy Science, Savoy, v. 77, p. 2738-2744, 1994.

BAILEY, C. M et al. Nulliparous versus primiparous crossbred females for beef. Journal of Animal Science, Savoy, v. 69, n. 4, p. 1403-1408, 1991.

BERGE, E.; WESTHUES, M. Técnica operatória veterinária. 5. ed. Montevideo: Labor S.A., 1975.

BROWN, J. R. Ovariectomizing Heifers. Modern Veterinary Practice, Wheaton, p. 13-15, 1984.

CROUSE, J. D. Comparisons of Bos indicus and Bos taurus inheritance on carcass beef characteristics and meat palatability. Journal of Animal Science, Savoy, v. 67, p. 2661, 1989.

DROST, M. et al. Ovariectomy by colpotomy in cows. Journal of Am. Vet. Med. Assoc., Schaumburg, v. 200, p. 337-339, 1992.

FARIAS JR., D. Efeitos da ovariectomia, aplicação de anel de látex no pedículo ovariano e dispositivo intra-uterino no ganho de peso, características de carcaça e carne em novilhas e vacas destinadas ao abate. Dissertação (Mestrado em Medicina Veterinária) - Faculdade de Ciências Agrárias e Veterinárias, Universidade Estadual Paulista, Jaboticabal, 2000.

FLECK, A. T. et al. Effect of growth 30 months on reproductive performance of beef heifers. Journal of Animal Science, Savoy, v. 51, n. 4, p. 816-821, 1980.

HABERMEHL, N. L. Heifer ovariectomy using the Willis spay instrument: technique, morbidity and mortality. Can. Vet. J., Ottawa, v. 34, p. 664-667, 1993. 
HILL, V. V. Spaying ranch heifers. Veterinary medicine/Small Animal Clinician, New York, p. 11091110, 1983.

HORTON, G. M. J. et al. Intravaginal devices for feedlot heifers. Journal of Animal Science, Savoy, v. 49, p. 915-917, 1979.

LYNCH, J. M. et al. Influence of timing of gain on growth and reproductive performance of beef replacement heifers. Journal of Animal Science, Savoy, v. 75, n. 7, p. 1715-1722, 1997.

O'BRIEN, C. A. et al. Effects of melengestrol acetate on the growth and reproductive physiology of fattening heifers. Journal of Animal Science, Savoy, v. 27, p. 3664-670, 1968.

PINEDA, M. H. Female reproductive system. In: McDONALD, L.E.; PINEDA, M.H. Veterinary endocrinology and reproduction, Philadelphia: Lea \& Febiger, 1989. 571 p.

REILING, B. A. et al. Effects of prenatal androgenization, melengestrol acetate, and Synovex-H on feedlot performance, carcass, and sensory tracts of once-calved heifers. J. Anim. Sci., Savoy, v. 74, n. 9 , p. 2043-2051, 1996.

SABA, N.; CUNNINGHAM, N. F.; MILLAR, P. G. Plasma progesterone, androstenidione and testosterone concentrations in heifers. Journal of Reproduction and Fertility, Birmingham, v. 45, n. 1, p. 37-45, 1975.

SILVA, H. L. et al. Efeitos da utilização do dispositivo intra-uterino (DIU) sobre o ganho de peso e o rendimento de carcaça de novilhas nelore destinadas ao abate. Ciência Animal Brasileira, Goiânia, v. 6, n. 2, p. 95-103, 2005.

SILVA, H. L. Efeitos da utilização do dispositivo intrauterino (DIU) sobre o ganho de peso e rendimento de carcaça de novilhas nelore destinadas ao abate. Dissertação (Mestrado em Medicina Veterinária) - Escola de Veterinária, Universidade Federal de Goiás, Goiás, 2001.

SILVA, L. A. F. et al. Descrição de duas técnicas cirúrgicas para castração de fêmeas bovinas e avaliação do pós-operatório. Cienc. Anim. Brás., Goiânia, v. 5, n. 2, 2004.

SILVA, L. A. F. et al. Anel de látex aplicado no pedículo ovariano de bezerras Nelore. Acta Sci. Anim. Sci., Maringá, v. 28, n. 1, p. 97-103, 2006.

TURIN, E. M. et al. Effects of a copper-bearing intrauterine device on the ovarian function, body weight gain and pregnancy rate of nulliparous heifers. Theriogenology, Montgomery, v. 47, p. 13271336, 1997.

YOUNG, A. W. et al. Effects on an oral progestogen on feedlot heifers. J. Anim. Sci. Savoy, v. 28, p. 224-227, 1969.

ZOBELL, R. D.; GOONEWARDENE, L. A.; ZIEGLER, C. B. K. The effects of spaying and anabolic implants on average daily weight gain of heifers on pasture. Can. Vet. J., Ottawa, v. 34, p. 727-730, 1993.

Recebido em: 19/12/2006

Received in: $12 / 19 / 2006$

Aprovado em: 30/03/2007

Approved in: 03/30/2007 